\title{
Inteligencias múltiples y aprendizaje de la lengua inglesa en estudiantes universitarios
}

\author{
Multiple Intelligences and English Language Learning in University Students
}

\author{
BETTY MARLENE LAVADO ROJAS ${ }^{1}$
}

https://orcid.org/0000-0002-2924-6771

\section{EDITH CONSUELO ZÁRATE ALIAGA}

https://orcid.org/0000-0003-1447-78731

\section{WALTER POMAHUACRE GÓMEZ ${ }^{3}$}

https://orcid.org/0000-0002-0276-9804

\begin{abstract}
RESUMEN: El objetivo fue establecer el grado de relación entre las inteligencias múltiples y el aprendizaje de la lengua inglesa en estudiantes del primer año de la Universidad Nacional de Educación Enrique Guzmán y Valle. Estudio de enfoque cuantitativo, tipo sustantiva, método descriptivo y diseño descriptivo-correlacional. La población estuvo conformada por 114 estudiantes; la muestra fue censal. Los datos fueron recogidos mediante un cuestionario adaptado del instrumento desarrollado por Howard Gardner y un test que incluye las cuatro habilidades básicas para el aprendizaje de la lengua inglesa: Listening, Speaking, Reading y Writing. Para establecer el grado de relación entre las variables en estudio, se aplicó el coeficiente de Correlación Chi Cuadrado y el de Rho de Spearman. Luego de su análisis, según la correlación de Spearman de 0,473, se llegó a la conclusión principal de que las inteligencias múltiples y el aprendizaje de la lengua inglesa se relacionan directamente con una correlación positiva moderada.
\end{abstract}

Palabras clave: aprendizaje; capacidades; habilidades; inteligencia; lengua extranjera.

ABSTRACT: This research had as aim to establish the degree of relationship between the multiple intelligences and the English language learning in students of the first year of the National University of Education Enrique Guzmán y Valle. It is a work confined to a quantitative approach, type substantive, correlational design and descriptive method. The population and the sample was consisted of 114 students. To gather the information, it was applied a questionnaire adapted from the one developed by Howard Gardner and a test, which includes the four basic skills for the English language learning: Listening, Speaking, Reading and Writing. With the purpose of establishing the degree of relationship between each of the variables of this study, it was applied the Coefficient of Correlation Chi-square 
and Rho Spearman. After their analysis, according to the Spearman correlation of 0,473, the main conclusion was reached that multiple intelligences and English Language Learning are directly related to a moderate positive correlation.

Keywords: learning; capacities; abilities; intelligence; foreign language.

\section{INTRODUCCIÓN}

Este trabajo tuvo como propósito general establecer el grado de correlación entre las inteligencias múltiples y el aprendizaje de la lengua inglesa en estudiantes del primer año de la Universidad Nacional de Educación Enrique Guzmán y Valle. Por ello, se consideró necesario describir las variables de estudio: las inteligencias múltiples y el aprendizaje de la lengua inglesa en el contexto de estudiantes universitarios.

Para describir la variable Inteligencias múltiples, fue necesario conocer las teorías que las sustentan. Al respecto, Gardner (1995) señaló que la inteligencia implica la habilidad necesaria para resolver un problema o para elaborar productos que son importantes en un contexto cultural y, sin negar que la inteligencia tiene numerosas propuestas, estas se encuentran entre dos corrientes, principalmente, unas que consideran que la inteligencia tiene una estructura unitaria; y otras, una estructura múltiple. Esta dicotomía se ha mantenido por mucho tiempo; pero, recientemente, estas posturas tienen un acercamiento. Carroll (1993) ha sistematizado esta idea y propone la existencia de una inteligencia compleja, organizada jerárquicamente. Sin embargo, subsisten modelos alternativos a este acuerdo y uno de ellos es la Teoría de las Inteligencias Múltiples propuesta por Howard Gardner. Según este autor, las capacidades de nuestra mente no forman parte de una sola habilidad llamada inteligencia, sino de muchas que trabajan en paralelo y que, generalmente, son ignoradas simplemente porque no las valoramos. Según la Teoría de las Inteligencias Múltiples, cada persona dispone de varios tipos de habilidades mentales que son independientes entre sí; por ello, los seres humanos somos capaces de responder a retos e incluso improvisar con resultados halagadores. Gardner rompe con el esquema tradicional de inteligencia, a la cual le otorga una gama de capacidades del ser humano y fundamenta su teoría en las ocho capacidades cognitivas humanas, que son: la lingüística, la lógico-matemática, la corporal-kinestésica, la musical, la visual-espacial, la interpersonal, la intrapersonal y la naturalista; aunque, sugiere que pueden existir otras inteligencias que aún no se han descubierto.

a) Inteligencia lingüística. Se refiere a la adecuada construcción de las oraciones, la utilización de las palabras de acuerdo con sus significados y sonidos, al igual que la utilización del lenguaje de conformidad con sus diversos usos. Por ejemplo, los poetas, novelistas, entre otros.

b) Inteligencia musical. Se refiere al uso adecuado del ritmo, melodía y tono en la construcción y apreciación musical. Por ejemplo, Beethoven, Shakira, etc.

c) Inteligencia lógico-matemática. Referida a la facilidad para manejar cadenas de razonamiento e identificar patrones de funcionamiento en la resolución de problemas. Por ejemplo, Einstein, (...), los ingenieros. 
d) Inteligencia kinestésica. Señala la capacidad para manejar el cuerpo en la realización de movimientos en función del espacio físico y para manejar objetos con destreza. Por ejemplo, Babe, (...), Pelé.

e) Inteligencia visual-espacial. Referida a la habilidad para manejar los espacios, planos, mapas, y a la capacidad para visualizar objetos desde perspectivas diferentes. Por ejemplo, Gasparov, (...), los arquitectos.

f) Inteligencia intrapersonal. Señala la capacidad que tiene una persona para conocer su mundo interno, es decir, sus propias y más íntimas emociones y sentimientos, así como sus propias fortalezas y debilidades. Por ejemplo, Gandhi, (...), Freud.

g) Inteligencia interpersonal. Muestra la habilidad para reconocer las emociones y sentimientos derivados de las relaciones entre las personas y sus grupos. Por ejemplo, Nelson Mandela, (...), los líderes.

h) Inteligencia naturalista. Se refiere la habilidad para discriminar y clasificar los organismos vivos existentes en la naturaleza. Estas personas se reconocen parte del ecosistema ambiental. Por ejemplo, Darwin, (...), los biólogos.

Además de estas inteligencias, Gardner (2000) se encuentra investigando dos probables nuevas inteligencias, es decir, aún son planteamientos hipotéticos: la inteligencia moral y la existencial.

i) Inteligencia moral. Referida a las capacidades presentes en algunas personas para discernir entre el bien y el mal, preocupadas por el respeto a la vida y a la convivencia humana.

j) Inteligencia existencial. Señala la sensibilidad por la existencia del ser humano, se muestra inquieto por reflexiones sobre la trascendencia humana, sobre alfa y omega.

Respecto de la variable Aprendizaje de lenguas extranjeras que, en las últimas décadas, sugiere un enfoque comunicativo, cuyo postulado fundamental es que el aprendizaje de una lengua extranjera debe ir dirigida a que los estudiantes consigan desarrollar competencias comunicativas; es decir, a la adquisición de una serie de destrezas que les permitan comunicarse en esa lengua en las situaciones más corrientes de la vida cotidiana. Palacios (2008, p.56) sugiere que el enfoque comunicativo contribuye al desarrollo de la competencia comunicativa del estudiante, quien logra una competencia lingüística, sociolingüística, habilidad para adecuar los medios lingüísticos a las características de cada contexto social, competencia discursiva que incluye la habilidad de analizar y sintetizar el texto para poder relacionarlo coherentemente en un texto parecido y exponerlo en público y, por último, la competencia estratégica que requiere la capacidad de iniciar, desarrollar y concluir la comunicación.

Las habilidades básicas que se desarrollan para el aprendizaje de la lengua inglesa son las que se detallan a continuación.

a) Comprensión auditiva (Listening). Tiene que ver con la "comprensión del habla oral" (Antich, 1986, p.209). Los estudiantes de la muestra deben desarrollar capacidades básicas en inglés, como entender frases y vocabulario frecuentes relaciona- 
dos con las áreas de importancia personal más inmediata (por ejemplo, información personal y de la familia muy básico, compras, área local, empleo). Los cuales se encuentran en mensajes y anuncios cortos, claros y simples.

a) Expresión oral (Speaking). Habilidad de expresar ideas, sentimientos, necesidades, deseos por medio del lenguaje, con fluidez y precisión. Los estudiantes de la muestra pueden describir información personal, así como sus experiencias más importantes (personales, de su familia, lugares de interés, ocupaciones, entre otros); pueden establecer contacto social con sus pares a la hora de realizar tareas simples y cotidianas.

b) Comprensión de lectura (Reading). Murcia (2000, p.58) plantea que la lectura implica la participación de un escritor, un texto y un lector; y este último debe ejecutar actividades como reconocer los signos escritos mediante la decodificación, interpretarlos y comprender la interacción del autor. En este contexto, los estudiantes de la muestra se encuentran en un nivel de inglés que pueden leer textos cortos y simples, encontrar la información específica, fiable en el material de uso diario como publicidad, prospectos, menús y horarios; además, pueden entender cartas personales cortas y sencillas.

a) Producción escrita (Writing). Los estudiantes deben desarrollar diversas capacidades para lograr la competencia escrita en el nivel de inglés en que se encuentran los estudiantes de la muestra, entre ellas, escribe notas y mensajes sencillos con información, instrucciones e indicaciones relacionadas con actividades de la vida cotidiana. Escribe correspondencia personal sencilla con la que puede dar las gracias, pedir disculpas, etc. Describe lugares o hechos de manera sencilla. Da coherencia al texto escrito, utilizando palabras y estructuras más importantes que indican orden temporal y causal.

Desde esa perspectiva, concordamos con Alarcón (2013), quien manifiesta que el desarrollo de las inteligencias múltiples en el aula favorece el aprendizaje del inglés y resulta motivador para los estudiantes. Del mismo modo, Palenzuela (2012) señala en su tesis que al aplicar un programa cuyo método se basa en las inteligencias múltiples, mejora la calidad de la enseñanza-aprendizaje del inglés en el adulto mayor. Al mismo tiempo, las inteligencias múltiples facilitan la atención a la diversidad en el aula, y abre un camino mediante el cual el aprendiente podrá descubrir sus propias debilidades y fortalezas y aprender a emplearlas para conseguir un aprendizaje efectivo dentro y fuera del aula. Por ello, no se puede negar que existe una relación positiva cuando el docente aplica actividades basadas en las inteligencias múltiples para el aprendizaje de la lengua inglesa, dada la variedad de capacidades que poseen los estudiantes y las que deben desarrollar para comunicarse efectivamente en el idioma.

\section{METODOLOGÍA}

La investigación se circunscribe en el enfoque cuantitativo. Al respecto, Monje (2011, p. 11) señala que el enfoque cuantitativo en el contexto de las ciencias sociales tiene el propósito de buscar solución a los fenómenos estableciendo regularidades en los mismos, esto es, hallar leyes generales que explican el comportamiento social. Respecto del tipo de investigación, es sustantiva. De acuerdo con Sánchez \& Reyes (2006), la investigación de tipo 
sustantiva es aquella que trata de responder a los problemas teoréticos o sustantivos, tal sentido, está orientada a describir, explicar, predecir o retrodecir la realidad, con lo cual se va en búsqueda de principios y leyes generales que permitan organizar una teoría científica. Dentro de este tipo de investigación se encuentra la descriptiva; por ello, sostenemos que nuestro trabajo de investigación se enmarca en este tipo.

Para realizar la investigación se optó por utilizar un diseño correlacional, que nos permitirá medir el grado de relación que existe entre las inteligencias múltiples y el aprendizaje del idioma inglés. El método que se utilizó en esta investigación fue el descriptivo. Para Sánchez \& Reyes (2009), este método consiste en describir, analizar e interpretar sistemáticamente un conjunto de hechos o fenómenos y sus variables que los caracterizan de manera tal como se da en el presente. Se describió y analizó las inteligencias múltiples y el aprendizaje de la lengua inglesa.

La población de estudio estuvo conformada por 114 estudiantes matriculados en el primer ciclo de estudios de las especialidades de Inglés-Italiano, Inglés-Español como Lengua Extranjera, Inglés-Francés e Inglés-Alemán, adscritos al Departamento Académico de Lenguas Extranjeras de la Universidad Nacional de Educación Enrique Guzmán y Valle. Por ser un estudio correlacional, se consideró como muestra a todas las unidades que conforman la población; por ello, la muestra fue de tipo censal.

Para el recojo de los datos necesarios para el análisis y la obtención de los resultados, se aplicó como técnica la encuesta y como instrumento para el recojo de la información sobre la variable Inteligencias múltiples, el cuestionario. Dicho instrumento incluyó 80 ítems, cada uno de los cuales tuvo cinco posibilidades de respuesta: totalmente en desacuerdo (1), en desacuerdo (2), ni de acuerdo ni desacuerdo (3), de acuerdo (4), Totalmente de acuerdo (5). Asimismo, el encuestado solo podía marcar una alternativa, con un aspa (X). Si marcaba más de una alternativa, se invalidaba el ítem. Además, su estructura estuvo distribuida entre las ocho dimensiones que correspondían a cada una de las inteligencias estudiadas: lingüística, lógico-matemática, visual-espacial, corporal-kinestésica, musical, intrapersonal, interpersonal y naturalista.

Para el recojo de datos de la variable Aprendizaje de la lengua inglesa, se aplicó el Test Cambridge English Movers, conocido también como Young Learners English, validado y aplicado por Cambridge University; además, es reconocido a escala internacional y refleja las destrezas lingüísticas y comunicativas del estudiante de la forma más fiable posible; ha sido diseñado para ser imparcial con los usuarios de todas las nacionalidades y orígenes lingüísticos y está respaldado por el mayor programa de investigación en su campo. El instrumento consta de cuatro partes, su escala es binomial. Asimismo, es necesario señalar que los niveles y rangos del examen aplicado para recoger la información sobre el aprendizaje de la lengua inglesa corresponden a esta variable y a cada una de las dimensiones: Muy baja [05], Baja [6-10], Regular [11-13], Alta [14-17] y Muy alta [18-20].

Las siguientes consideraciones éticas estuvieron presentes en el desarrollo de esta investigación: El derecho de autor, redactando las citas y referencias donde corresponda y de manera fidedigna. Como se recogió la información en una única vez, los docentes que aplicaron dichos instrumentos explicaron claramente a los sujetos los objetivos de la investigación. Además, se señaló a los sujetos que las respuestas eran confidenciales y no se 
publicarían sus datos personales y, para su consentimiento, se les solicitó su firma en una ficha adicional, en la que se incluyó sus datos personales y un código, que coincidió con el que aparecía en los instrumentos. Finalmente, se explicó a los sujetos que el resultado de esta investigación será utilizado para proponer la metodología y las estrategias que se deberían implementar en los sílabos de las distintas asignaturas de su carrera, con el fin de mejorar el nivel de logro de cada uno de ellos.

\section{RESULTADOS}

\section{Análisis descriptivo de los resultados obtenidos de la variable Inteligencias Múltiples}

De acuerdo con el análisis descriptivo de cada una de las variables y dimensiones, podemos interpretar que para la variable Inteligencias Múltiples, los estudiantes del primer año de las especialidades de lenguas extranjeras de la Universidad Nacional de Educación Enrique Guzmán y Valle evidencian que tienen un nivel de desarrollo de sus inteligencias que va de Medio a Muy alto y que equivale al 88,6 \%, siendo el rango Medio el que obtuvo el mayor porcentaje 50,9\%, frente al 11, $4 \%$ que se ubica en un nivel Bajo. Lo manifestado se apoya en la definición de Gardner (1983), quien señala que el ser humano posee varios tipos de habilidades mentales y que son independientes entre sí.

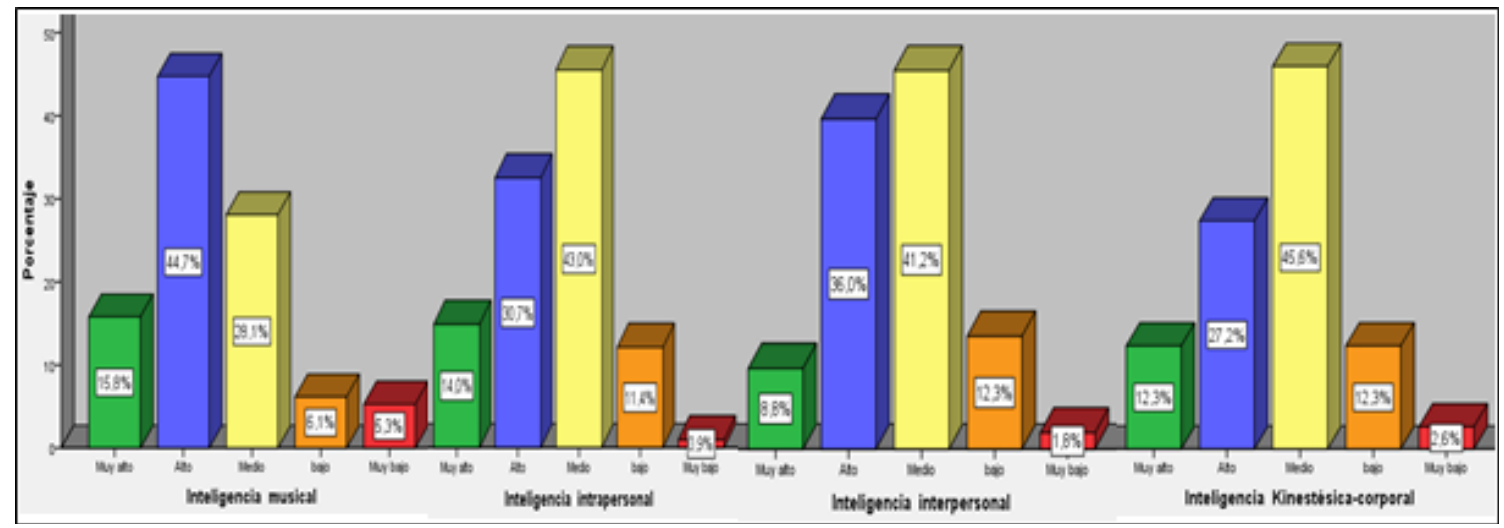

Figura 1. Resultados obtenidos en las inteligencias musical, intrapersonal, interpersonal y corporal-kinestésica

Como se muestra en la figura 1, la inteligencia musical es la que obtuvo el mayor nivel, considerando los rangos Medio, Ato y Muy alto, alcanzando el 88,6 \%, siendo el rango Alto el que muestra el mayor porcentaje (44,7); frente a ello, el 11,4\% de estudiantes posee nivel Bajo y Muy bajo. Este resultado nos permite afirmar que los estudiantes han desarrollado el hemisferio derecho donde se localiza la percepción y producción musical. Por ello, poseen capacidad para escuchar, cantar, crear piezas musicales, entre otras (Mercadé, A., 2008, p.4).

Se evidencia que la inteligencia intrapersonal alcanzó el 87,7 \%, siendo el rango Medio el que tiene el mayor porcentaje (43,0 \%). Y, un 12,3\% de estudiantes que poseen nivel Bajo y Muy bajo en esta inteligencia. Lo cual quiere decir que los estudiantes son capaces de 
analizar lo que ocurre en sus mentes y les permite la capacidad de acceder a sus propios sentimientos y emociones que guían su comportamiento y su conducta. Según Mercadé (2008, p.5), las capacidades implicadas son aquellas que les permiten trazarse metas, evaluar sus habilidades y desventajas personales, controlar su pensamiento propio. Los estudiantes que desarrollan esta habilidad en mayor grado son capaces de escribir autobiografías, diarios, reflexiones, ensayos, elaboran portafolios con poemas, canciones, etcétera.

La inteligencia interpersonal ocupa el tercer lugar, cuyo rango Medio es de mayor porcentaje $(41,2)$ y muestra un $86 \%$ de estudiantes que desarrollan esta inteligencia en los rangos Muy alto, Alto y Medio; frente al 14,1 que se ubican en un nivel Bajo y Muy bajo. Lo cual nos permite afirmar que los estudiantes tienen buenas relaciones entre pares, comprenden las emociones y el comportamiento de sus compañeros; por ello, son capaces de trabajar de manera cooperativa y colaborativa, proponer y ejecutar proyectos en clase y fuera de ella, gustan de realizar diálogos y conversaciones, juegos de roles, componer canciones e historias de manera grupal, entre otros.

Luego se ubica la inteligencia corporal-kinestésica, en la que se aprecia que también el rango Medio es mayor (45,6); sin embargo, se aprecia que el 85,1\% de estudiantes se ubica en los rangos Muy alto, Alto y Medio, frente al 14,9\% que se ubica en el nivel Bajo y Muy bajo. Según Mercadé (2008, p.4), las capacidades implicadas son aquellas que permiten a los estudiantes realizar actividades que requieren fuerza, rapidez, flexibilidad, coordinación óculo-manual y equilibrio; desarrollan habilidades manuales para crear o hacer reparaciones; y son muy buenos expresándose con el cuerpo. Estas habilidades son útiles al momento de aprender un idioma a través de la actuación de historias, diseño de accesorios, participación en juegos, elaboración de diversos objetos para aprender, por ejemplo, vocabulario, entre otros.

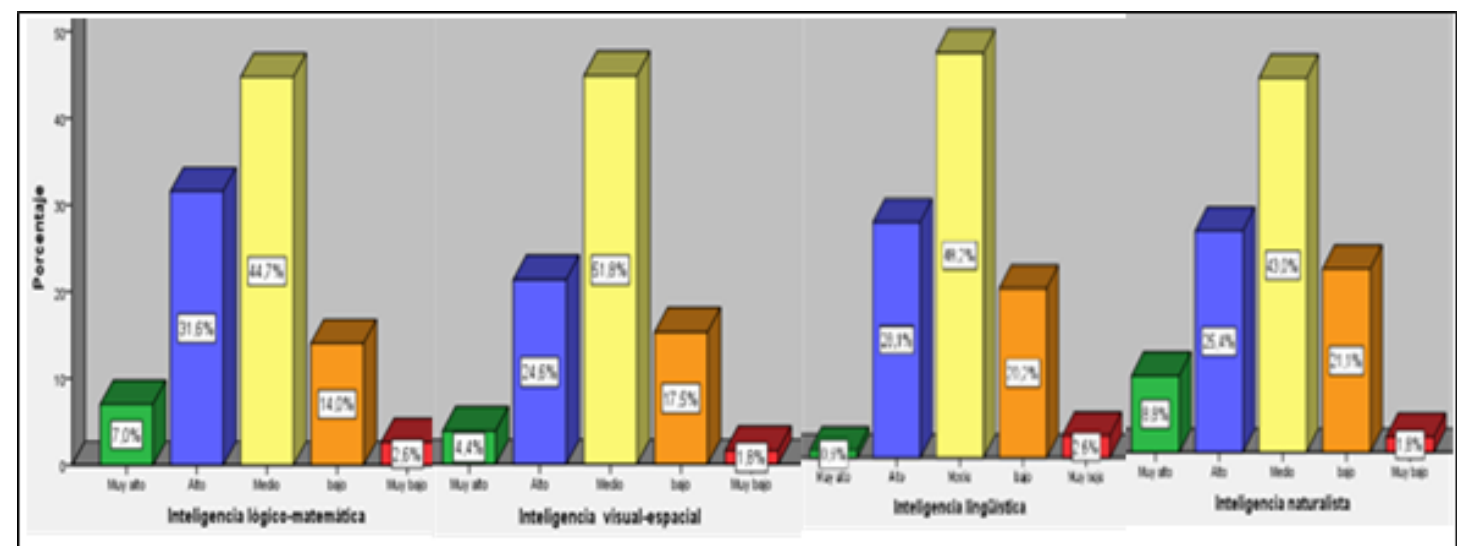

Figura 2. Resultados obtenidos en las inteligencias lógico-matemática, visual-espacial, lingüística y naturalista

Como se muestra en la figura 2, la inteligencia lógico-matemática ocupa el quinto lugar, y el 83,3\% de estudiantes tienen este tipo de inteligencia, siendo el rango Medio el de mayor nivel (44,7), y un 16,6 \% se ubica en el nivel Bajo y Muy bajo. A decir de Perea (2017), esta inteligencia consiste en promover el desarrollo de estrategias de razonamiento. Para trabajar esta inteligencia, son útiles los números y la resolución de problemas numéricos. Asimismo, al leer un problema varias veces para resolverlo, los estudiantes se van familiarizando con el vocabulario y las estructuras utilizadas. Es decir, permite la habilidad de 
resolver operaciones mentales, resolución de problemas, entre otros. Podemos acotar que los estudiantes de lenguas extranjeras que poseen esta inteligencia lógico-matemática con mayor desarrollo que otras aprenden con facilidad las estructuras gramaticales y se familiarizan con ellas.

La inteligencia visual-espacial evidenció que un 80,8 \% de estudiantes se ubican en los rangos de Medio a Muy alto, siendo el rango Medio el de mayor porcentaje $(51,8)$, el 19,3 $\%$ se ubica en el nivel Bajo y Muy bajo. Según Mercadé (2008, p.3), los estudiantes poseen capacidades para presentar ideas visualmente, crear imágenes mentales, percibir detalles visuales, dibujar y confeccionar bocetos. Por ello, las actividades que pueden realizarse para el aprendizaje de un idioma son: tomar fotografías y hacer comentarios al respecto, utilizar organizadores gráficos, mapas mentales, ubicar lugares, describir utilizando líneas de tiempo, entre otras.

La inteligencia lingüística se ubica en el sétimo lugar, con el 77,3\% que se encuentran en los rangos de Medio a Muy alto, siendo el rango Medio el que ocupa el mayor porcentaje $(48,2)$. Se puede apreciar que el 22,8 \% tiene un nivel Bajo y Muy bajo de desarrollo de la inteligencia lingüística. Para Mercadé (2008, p.3), los estudiantes desarrollan la capacidad para comprender el orden y el significado de las palabras en la lectura, la escritura y, también, al hablar y escuchar; lo cual permite escribir adecuadamente. Por lo que Perea (2017) señala que esta inteligencia se utilizará para incentivar la comunicación y la interacción. Además, en las etapas iniciales del aprendizaje, es más importante el desarrollo de competencias orales en la enseñanza-aprendizaje del inglés con enfoque comunicativo. Por ello, es muy importante el desarrollo de actividades como: Lectura de historias cortas, poemas, descripciones, narraciones, periódicos. Fomentar los debates en grupos o de manera individual, declamación de poesías cortas, improvisación de discursos sobre temas de interés de los estudiantes, sobre las actividades diarias. Memorización de poemas, canciones o historias. Contar chistes o decir adivinanzas en la clase. Desarrollar crucigramas y otros juegos de palabras, entre otras.

La inteligencia naturalista ocupó el último lugar; no obstante, los estudiantes demostraron un porcentaje apreciable de desarrollo de esta inteligencia. Así, apreciamos que el 77,2 \% se ubica en los rangos de Medio a Muy alto, siendo el de mayor porcentaje el rango Medio $(43,0)$; y, el 22,9 \% poseen un nivel Bajo y Muy bajo de desarrollo de la inteligencia naturalista. Los estudiantes desarrollan la habilidad de observación, experimentación, reflexión, cuestionamiento de nuestro entorno; por ello, desarrollan la sensibilidad para convivir en su ambiente y promover una cultura de paz, de respeto al entorno. Actividades como observar el ambiente natural que los rodea y hacer descripciones, escribir reflexiones acerca de cómo conservar el ambiente, escribir o exponer acerca de la convivencia con la naturaleza y con otros seres, describir a sus mascotas, entre otras, son útiles para el aprendizaje de la lengua inglesa.

\section{Análisis descriptivo de los resultados obtenidos de la variable Aprendizaje de la Lengua Inglesa}

En lo que respecta a la variable Aprendizaje de la lengua inglesa, se puede señalar que los estudiantes de lenguas extranjeras del primer año de estudios tienen un nivel Alto, con el 49,1\%, seguido del $19 \%$ de estudiantes que tiene un nivel Medio y el 18,4 \% que evidencia 
un nivel Muy alto, lo que nos permite señalar que es mayor el porcentaje de estudiantes con dominio de la lengua inglesa, frente al 13,2 \% que muestra un nivel Bajo en el aprendizaje de dicha lengua.

Al analizar los resultados obtenidos en cada una de las dimensiones del aprendizaje de la lengua inglesa, se puede apreciar que la dimensión Comprensión auditiva (Listening) obtuvo el menor porcentaje entre los estudiantes del primer año; siendo el nivel Alto, el que ocupó el mayor porcentaje con un 35,1 \%, seguido del nivel Muy alto, con el 22,8 \% y el nivel Medio con un 14,9 \%; frente al nivel Bajo, que fue de 21,9\% y nivel Muy bajo con el $5,3 \%$. Esta habilidad es esencial y básica en el aprendizaje de una lengua y permite el desarrollo de capacidades básicas, como: entender lo que se dice en una conversación cotidiana sencilla, reconocer el tema de conversación, comprender frases y expresiones de uso diario, comprender el sentido de anuncios, mensajes públicos breves y sencillos, comprender noticias con ayuda de imágenes, etcétera.

La expresión oral los estudiantes alcanza un nivel Muy alto en un 35,1\%, seguido del nivel Alto que tiene el 33,3 \% y el nivel Medio donde obtuvieron el 11,4 \%; mientras que el 15,8 $\%$ se encuentran en un nivel Bajo y el 4,4 \% en un nivel Muy bajo. Estos resultados nos permiten señalar que los estudiantes se expresan en lengua inglesa, y pueden realizar diversas actividades, desde los aspectos de interacción y producción; es decir, comunicarse con otros y expresarse en situaciones planteadas. Por ello, podemos indicar que los estudiantes están en condiciones de describir, de manera sencilla, información personal, experiencias importantes acerca de ellos, su familia, lugares de interés, ocupaciones, entre otros. Además, están capacitados para establecer contacto social con sus pares al momento de realizar tareas habituales, con intercambio sencillo de información. Pueden dar información concreta de cómo llegar a algún lugar, etcétera.

La dimensión Comprensión de lectura presenta los mismos niveles y rangos que la dimensión anterior. Los estudiantes alcanzaron en el nivel Muy alto un 35,1 \%, seguido del nivel Alto que tiene el 33,3 \% y el nivel Medio donde obtuvieron el 11,4\%; mientras que el $15,8 \%$ se encuentran en un nivel Bajo y el 4,4 \% en un nivel Muy bajo. Según el MCER, la lectura es una habilidad de comprensión y los estudiantes deben comprender aspectos sencillos y de uso diario. Al respecto, debemos señalar que los estudiantes demostraron competencia de comprensión de lectura, por lo que han desarrollado las siguientes capacidades: Comprende notas y mensajes breves relacionados con la vida personal y cotidiana, comprende textos breves y sencillos sobre cuestiones prácticas, entre ellas, reservar una habitación, información sobre un curso en el extranjero, etc.; comprende instrucciones e indicaciones utilizadas en la vida diaria, identifica y comprende información en folletos y otros materiales informativos, así como información relevante en textos breves y sencillos, comprende narraciones o relatos breves escritos de manera sencilla.

En lo que respecta a la dimensión Producción escrita de la lengua inglesa, se puede apreciar que los estudiantes demuestran un nivel Alto que equivale al 37,7 \% y Muy Alto, con el 23,3 \%, siendo el nivel Medio el que ocupa el tercer lugar con el 17,5\%; mientras que un 14 $\%$ tiene un nivel Bajo y un $4,4 \%$ un nivel muy bajo. Al respecto, el MCER señala que los estudiantes pueden escribir una postal corta y simple, por ejemplo, enviar saludos de vacaciones. Ellos pueden llenar formatos con información personal, por ejemplo, escribiendo su nombre, nacionalidad y registrarse en un formato de un hotel, entre otras actividades. Habi- 
tualmente, se deja esta habilidad para ser desarrollada al final; sin embargo, es importante señalar que no puede ir aislada, porque existe una relación estrecha entre la lengua oral y escrita, por ende, ambas deben integrarse para un mejor desarrollo.

Los resultados encontrados nos permiten señalar que las inteligencias múltiples son un soporte para el aprendizaje de la lengua inglesa y de cualquier otra que se desee aprender. Además, las actividades presentadas en el aula de clases pueden integrarse, de modo que se permita a todos los estudiantes desarrollar las diversas inteligencias $\mathrm{y}$, de esa manera, mejorar el aprendizaje de la lengua.

\section{DISCUSIÓN}

Como el objetivo general de la investigación proponía establecer el grado de relación entre las inteligencias múltiples y el aprendizaje de la lengua inglesa en los estudiantes del primer año de la Universidad Nacional de Educación Enrique Guzmán y Valle, 2018, la prueba de hipótesis general demostró que existe una relación directa entre las variables de estudio. Según la prueba de la independencia Chi-cuadrado: 16,919 y con 9 grados de libertad, presentó un valor de significancia de 0,000 menor a 0,05; es decir, significativo. Asimismo, según la correlación de Spearman de 0,473, representó esta una correlación positiva moderada; por lo que se puede manifestar que las inteligencias múltiples están relacionadas directamente con el aprendizaje de la lengua inglesa; por ello, podemos decir que en cuanto mayor sea el conocimiento del uso de las inteligencias múltiples, existirán mayores niveles de aprendizaje de la lengua inglesa. Al respecto, Alarcón (2013) señaló que el desarrollo de las inteligencias múltiples en el aula favorece el aprendizaje del inglés y resultó motivador para los estudiantes. La realización de actividades enfocadas a cubrir las inteligencias múltiples elegidas para este estudio ha favorecido el aprendizaje del inglés por parte de los estudiantes que se han mostrado entusiasmados y dispuestos a participar, demandando más actividades de este tipo. Del mismo modo, Palenzuela (2012) afirmó que los efectos del método basado en inteligencias múltiples del estudiante son muy positivos para el adulto mayor, ya que este necesitó grandes dosis de motivación y estímulo; y el método implementado pudo proporcionarle debido a las características del mismo. Esto tuvo como consecuencia una participación activa y un elevado grado de implicación por parte del estudiante en el aprendizaje del inglés. Sin embargo, Bartolomei, \& Aguaded (2018) señalaron que no se pudo comprobar que la aplicación de estrategias, que incluye el uso de las inteligencias múltiples, puede mejorar el proceso de enseñanza aprendizaje de lenguas extranjeras. Además, no encontró diferencias significativas respecto del grupo experimental del estudio y el grupo control. Asimismo, Luengo (2015) señaló que las preferencias en cuanto a estilo de aprendizaje o inteligencia múltiple en el aprendizaje contribuyeron poco a explicar el rendimiento; además, sustentó su resultado en lo manifestado por Martín \& Mauri (2011), quienes afirmaron que es un resultado positivo que las variables de Estilos de aprendizaje o Inteligencias múltiples no predigan el posible rendimiento, toda vez que esto quiere decir que el proceso de enseñanza-aprendizaje es equitativo y no hay preferencias que queden favorecidas.

El objetivo específico $\mathrm{n}^{\circ} 1$ pretendía establecer el grado de relación entre la inteligencia lingüística y el aprendizaje de la lengua inglesa; la prueba de hipótesis permitió probar que 
existe relación directa entre la dimensión inteligencia lingüística y el aprendizaje de la lengua inglesa. Y, según la correlación de Spearman de 0,508, representó esta una correlación positiva moderada; por lo que se pudo señalar que la inteligencia lingüística está relacionada directamente con el aprendizaje de la lengua inglesa, es decir, cuanto mayor sea el conocimiento del uso de la inteligencia lingüística, existirán mayores niveles de aprendizaje de la lengua inglesa. Al respecto, Arias \& Llamozas (2011) señalaron que la relación entre la inteligencia verbal y el nivel de logro del aprendizaje del idioma inglés como segunda lengua es moderada, es decir que no se encontró un nivel alto de significancia entre ambas variables. Del mismo modo, Luengo (2015) indicó que las inteligencias lingüística y musical suelen tener relación significativa positiva con el rendimiento, por lo que se cumple la hipótesis inicial de la relación significativa de la inteligencia lingüística con el rendimiento.

El objetivo específico $\mathrm{n}^{\circ} 2$ tuvo el propósito de establecer el grado de relación entre la inteligencia lógico-matemática y el aprendizaje de la lengua inglesa, y la prueba de hipótesis permitió demostrar que existe relación directa entre la dimensión inteligencia lógico-matemática y el aprendizaje de la lengua inglesa. Asimismo, según la correlación de Spearman de 0,416 , representó esta una correlación positiva moderada, por lo que se pudo inferir que la inteligencia lógico-matemática está relacionada directamente con el aprendizaje de la lengua inglesa; por ello, cuanto mayor sea el conocimiento del uso de la inteligencia lógico-matemática, existirán mayores niveles de aprendizaje de la lengua inglesa. Al respecto, Inga, Basilio, \& Peña (2017) comprobaron que la variable inteligencia lógico-matemática tiene correlación positiva con el rendimiento académico en estudiantes de la Facultad de Ingeniería Civil de la UNCP, lo que implica que a mayor nivel de inteligencia lógico-matemática, mayor será el rendimiento académico. Sin embargo, difiere de lo hallado por Luengo (2015), pues ella manifiesta que la inteligencia lógico-matemática no resulta significativa en relación con el rendimiento.

El objetivo específico $n^{\circ} 3$ proponía establecer el grado de relación entre la inteligencia visual-espacial y el aprendizaje de la lengua inglesa. En la prueba de hipótesis se pudo comprobar que existe relación directa entre la dimensión inteligencia visual-espacial y el aprendizaje de la lengua inglesa. Asimismo, según la correlación de Spearman de 0,276, representó esta una correlación positiva Muy baja; sin embargo, dada esta correlación positiva baja, la inteligencia visual-espacial está relacionada directamente con el aprendizaje de la lengua inglesa; por ello, cuanto mayor sea el conocimiento del uso de la inteligencia visual-espacial, existirán mayores niveles de aprendizaje de la lengua inglesa. Al respecto, Luengo (2015) manifestó que las inteligencias visual-espacial y naturalista, siempre que aparecen en relaciones significativas, son negativas.

El objetivo específico $\mathrm{n}^{\circ} 4$ se propuso establecer el grado de relación entre la inteligencia corporal-kinestésica y el aprendizaje de la lengua inglesa. En la prueba de hipótesis, se observó que existe relación directa entre ambas variables. Asimismo, según la correlación de Spearman de 0,353, representó esta una correlación positiva baja. Sin embargo, se pudo señalar que, a pesar de esta correlación positiva baja, la inteligencia corporal-kinestésica está relacionada directamente con el aprendizaje de la lengua inglesa; es decir, cuanto mayor sea el conocimiento del uso de la inteligencia corporal-kinestésica, existirán mayores niveles de aprendizaje de la lengua inglesa. Al respecto, Alarcón (2013) destacó la óptima respuesta del grupo de discentes hacia las actividades planteadas, en las que participaron activamente 
demostrando ser muy despiertos y creativos, sorprendiendo con divertidas ocurrencias relacionadas con la situación comunicativa. Por otro lado, este grupo, en general, es muy dinámico; incluso, agradeció este tipo de actividades en las que el movimiento estaba presente, fomentándose la inteligencia corporal-kinestésica. A propósito, Luengo (2015) señaló que la inteligencia corporal-kinestésica aparece tanto con relación positiva como negativa dependiendo del curso. Asimismo, Carpio (2014) manifestó que el nivel de desarrollo de la inteligencia corporal-kinestésica, según la percepción de los padres, es Medio alto, según la percepción de los niños, es Bajo, Medio bajo y Medio y, según la percepción del docente, es Medio.

El objetivo específico $n^{\circ} 5$ pretendía establecer el grado de relación entre la inteligencia musical y el aprendizaje de la lengua inglesa. La prueba de hipótesis demostró que existe relación directa entre la dimensión inteligencia musical y el aprendizaje de la lengua inglesa. Asimismo, según la correlación de Spearman de 0,471, representó esta una correlación positiva moderada. Por lo tanto, se pudo inferir que la inteligencia musical está relacionada directamente con el aprendizaje de la lengua inglesa; por ello, cuanto mayor sea el conocimiento del uso de la inteligencia musical, existirán mayores niveles de aprendizaje de la lengua inglesa. Al respecto, Carpio (2014) señaló que el nivel de desarrollo de la inteligencia musical, según la percepción de los padres, es Medio alto, según la percepción de los niños, es Medio bajo y, según la percepción del docente, es Medio. Del mismo modo, Luengo (2015) manifestó que las inteligencias lingüística y musical suelen tener relación significativa positiva con el rendimiento.

El objetivo específico ${ }^{\circ} 6$ que proponía establecer el grado de relación entre la inteligencia intrapersonal y el aprendizaje de la lengua inglesa demostró, en la prueba de hipótesis, que existe relación directa entre la dimensión inteligencia intrapersonal y el aprendizaje de la lengua inglesa. Asimismo, según la correlación de Spearman de 0,469, representó esta una correlación positiva moderada. Por lo tanto, se pudo señalar que la inteligencia intrapersonal está relacionada directamente con el aprendizaje de la lengua inglesa; por ello, cuanto mayor sea el conocimiento del uso de la inteligencia intrapersonal, existirán mayores niveles de aprendizaje de la lengua inglesa. En relación con el resultado encontrado, se pudo comparar con el hallado por Alarcón (2013), quien manifestó que al analizar los cuestionarios ha resultado muy significativo el importante papel de la inteligencia emocional: interpersonal e intrapersonal. Al respecto, Carpio (2014) estableció en su investigación que el nivel de desarrollo de la inteligencia Intrapersonal, según la percepción de los padres, es Alto; según la percepción de los niños, es Medio alto; y, según la percepción del docente, es Medio alto. Asimismo, Ramiro (2017) señaló que las inteligencias más desarrolladas y por las cuales el estudiante ha sido capaz de utilizar la segunda lengua extranjera para desarrollar el propio proceso de enseñanza-aprendizaje son la inteligencia lingüística, corporal-kinestésica, natural, interpersonal e intrapersonal.

El objetivo específico $\mathrm{n}^{\circ} 7$ que se propuso establecer el grado de relación entre la inteligencia interpersonal y el aprendizaje de la lengua inglesa observó, en la prueba de hipótesis, que existe relación directa entre la dimensión inteligencia interpersonal y el aprendizaje de la lengua inglesa. Asimismo, según la correlación de Spearman de 0,399, representó esta una correlación positiva moderada. Por lo tanto, se pudo inferir que la inteligencia interpersonal está relacionada directamente con el aprendizaje de la lengua inglesa; por ello, cuanto 
mayor sea el conocimiento del uso de la inteligencia interpersonal, existirán mayores niveles de aprendizaje de la lengua inglesa. Al respecto, Carpio (2014) estableció en su investigación que el nivel de desarrollo de la inteligencia Interpersonal, según la percepción de los padres, es Alto; según la percepción de los niños, es Medio bajo; y, según la percepción del docente, es Medio. Al respecto, Torres y Valdivieso (2013) señalaron que, de acuerdo con las aplicaciones realizadas en el grado del Colegio de Bachillerato Universidad Libre, se pudo interpretar que las tareas que iban enfocadas a trabajar la inteligencia interpersonal propiciaron mayor desempeño en la competencia comunicativa de los estudiantes que aquellas tareas que apuntaban a un trabajo enfocado en la inteligencia intrapersonal, debido a que los estudiantes pueden suplir sus falencias a través de las fortalezas que poseen los demás integrantes del equipo. Del mismo modo, Alarcón (2013) manifestó que al analizar los cuestionarios ha resultado muy significativo el importante papel de la inteligencia emocional: interpersonal e intrapersonal. El grupo investigado destacaba por un alto nivel de inteligencia interpersonal, lo que le permitió sacar el máximo partido a las actividades propuestas, creándose un ambiente muy propicio para el aprendizaje del inglés.

El objetivo específico $n^{\circ} 8$ que se propuso establecer el grado de relación entre la inteligencia naturalista y el aprendizaje de la lengua inglesa observó, en la prueba de hipótesis, que existe relación directa entre la dimensión inteligencia naturalista y aprendizaje de la lengua inglesa. Asimismo, según la correlación de Spearman de 0,308, representó esta una correlación positiva moderada. Por lo tanto, se pudo inferir que la inteligencia naturalista está relacionada directamente con el aprendizaje de la lengua inglesa; por ello, cuanto mayor sea el conocimiento del uso de la inteligencia naturalista, existirán mayores niveles de aprendizaje de la lengua inglesa. Al respecto, Carpio (2014) estableció en su investigación que el nivel de desarrollo de la inteligencia naturalista, según la percepción de los padres, es Alto; según la percepción de los niños, es Medio; y, según la percepción del docente, es Medio. Del mismo modo, Ramiro (2017) señaló que las inteligencias más desarrolladas y por las cuales el estudiante ha sido capaz de utilizar la segunda lengua extranjera para desarrollar el propio proceso de enseñanza-aprendizaje son la inteligencia lingüística, corporal-kinestésico, natural, interpersonal e intrapersonal.

\section{CONCLUSIONES}

La conclusión general a la se arribó luego de la prueba de hipótesis fue que las inteligencias múltiples y el aprendizaje de la lengua inglesa en los estudiantes del primer año de la Universidad Nacional de Educación Enrique Guzmán y Valle presentan una correlación positiva moderada. Es decir, las inteligencias múltiples están relacionadas directamente con el aprendizaje de la lengua inglesa; por ello, en cuanto mayor sea el conocimiento del uso de las inteligencias múltiples, existirán mayores niveles de aprendizaje de la lengua inglesa.

La inteligencia lingüística y el aprendizaje de la lengua inglesa en los estudiantes de la muestra presentan una correlación positiva moderada. Es decir, la inteligencia lingüística se relaciona directamente con el aprendizaje de la lengua inglesa; por ello, se puede señalar que los estudiantes que desarrollan este tipo de inteligencia tendrán también mayores niveles de aprendizaje de la lengua inglesa. 
La inteligencia lógico-matemática y el aprendizaje de la lengua inglesa en los estudiantes de la muestra presentan una correlación positiva moderada. Es decir, la inteligencia lógico-matemática está relacionada directamente con el aprendizaje de la lengua inglesa; por ello, si los estudiantes desarrollan este tipo de inteligencia, pueden lograr un mayor nivel de aprendizaje de la lengua inglesa.

La inteligencia visual-espacial y el aprendizaje de la lengua inglesa en los estudiantes de la muestra presentan una correlación positiva muy baja. Sin embargo, se puede decir que un estudiante que desarrolla este tipo de inteligencia también podrá lograr un nivel de aprendizaje del idioma inglés.

La inteligencia corporal-kinestésica y el aprendizaje de la lengua inglesa en los estudiantes de la muestra también evidencian una correlación positiva baja. Sin embargo, se puede afirmar que un estudiante que desarrolle este tipo de inteligencia puede lograr un nivel de aprendizaje de la lengua inglesa.

La inteligencia musical y el aprendizaje de la lengua inglesa en los estudiantes de la muestra representan una correlación positiva moderada. Es decir, la inteligencia musical está relacionada directamente con el aprendizaje de la lengua inglesa; por ello, en cuanto mejor sea la inteligencia musical, existirán mayores niveles de aprendizaje de la lengua inglesa.

La inteligencia intrapersonal y el aprendizaje de la lengua inglesa en los estudiantes de la muestra presentan una correlación positiva moderada. Es decir, la inteligencia intrapersonal está relacionada directamente con el aprendizaje de la lengua inglesa; por ello, un estudiante que desarrolle este tipo de aprendizaje también logrará un mayor nivel de aprendizaje de la lengua inglesa.

La inteligencia interpersonal y el aprendizaje de la lengua inglesa en los estudiantes de la muestra evidencian una correlación positiva moderada. Es decir, la inteligencia interpersonal está relacionada directamente con el aprendizaje de la lengua inglesa; por ello, en cuanto mejor sea la inteligencia interpersonal, existirán mayores niveles de aprendizaje de la lengua inglesa.

La inteligencia naturalista y el aprendizaje de la lengua inglesa en los estudiantes de la muestra presentan una correlación positiva moderada. Es decir, la inteligencia naturalista está relacionada directamente con el aprendizaje de la lengua inglesa; por ello, si el estudiante desarrolla este tipo de inteligencia, logrará un mayor nivel de aprendizaje de la lengua inglesa.

\section{REFERENCIAS}

Alarcón, A. (2013). Las inteligencias múltiples en el aula de lengua inglesa [Tesis de máster, Universidad de Almería]. Repositorio Institucional de la Universidad de Almería. https://core.ac.uk/reader/143456570

Antich de León, R. (1986). Metodología de la enseñanza de las Lenguas Extranjeras. Editorial Pueblo y Educación. 
Arias, W. L., \& Llamosas, L. G. (2011). Inteligencia verbal y nivel de logro del aprendizaje del inglés como segunda lengua. Revista Iberoamericana De Educación, 55(1), 1-10. https://doi.org/10.35362/rie5511620

Bartolomei, P., \& Aguaded, E. M. (2018). Aplicación e impacto de las Inteligencias Múltiples en la Enseñanza de Lenguas Extranjeras. Revista Electrónica Interuniversitaria de Formación del Profesorado, 21(1), 95-114. https://doi.org/10.6018/reifop.21.1.281841

Carpio, L. (2014). Evaluación de las inteligencias múltiples en los estudiantes del $6^{\circ}$ año de educación general básica [Tesis de Bachillerato, Universidad de Cuenca]. Repositorio Institucional de la Universidad de Cuenca. http://dspace.ucuenca.edu.ec/ handle/123456789/20951

Carroll, J. B. (1993). Human cognitive abilities: A survey of factor-analytic studies. Cambridge University Press.

Common European Framework of Reference for Languages: Learning, teaching, assessment. The English edition is published by Cambridge University Press.

Gardner, H. (1987). Arte, Mente y Cerebro. Paidós.

Gardner, H. (1988). La nueva ciencia de la mente. Paidós.

Gardner, H. (1993). La mente no escolarizada. Paidós.

Gardner, H. (1993). Multiple intelligences: The theory in practice. Basic Books.

Gardner, H. (1994). Estructuras de la mente. Fondo de Cultura Económica.

Gardner, H. (1995). Inteligencias Múltiples. La Teoría en la Práctica. Paidós.

Gardner, H. (1995). Mentes Creativas. Una anatomía de la creatividad humana. Paidós.

Gardner, H. (1998). Mentes Extraordinarias: Cuatro retratos para descubrir nuestra propia excepcionalidad. Paidós.

Gardner, H. (1999). Intelligence reframed. Multiple intelligences for the 21st. Century. Basic Books.

Gardner, H. (2000). La educación de la mente y el conocimiento de las disciplinas: Lo que todo estudiante debería aprender. Paidós.

Gardner, H. (2001). La inteligencia reformulada. Las inteligencias múltiples en el siglo $X X I$. Paidós.

Inga, R. y Basilio, H. y Peña, P. (2017). Inteligencia lógico matemático y rendimiento académico en estudiantes de la Facultad Ingeniería Civil - UNCP. Horizonte de la Ciencia. 7. 10.26490/uncp.horizonteciencia.2017.13.360.

Inga, R., Basilio, H., \& Peña, P. (2017). Inteligencia lógico matemático y rendimiento académico en estudiantes de la Facultad Ingeniería Civil - UNCP. Horizonte De La Ciencia, 7(13), 139-148. Recuperado a partir de http://revistas.uncp.edu.pe/index. $\mathrm{php} /$ horizontedelaciencia/article/view/322

Luengo, E. (2015). Análisis de estilos de aprendizaje e inteligencias múltiples de futuros maestros en relación al aprendizaje de una lengua extranjera [Tesis de Doctorado, 
Universidad Nacional de Educación a Distancia]. http://e-spacio.uned.es/fez/eserv/ tesisuned:Educacion-Eluengo/LUENGO_CERVERA_Esperanza_Tesis.pdf

Martín, E. y Mauri, T. (Coords.) (2011). Orientación Educativa. Atención a la diversidad y educación inclusiva. Graó.

Mercadé, A. (2008). Los 8 tipos de Inteligencia según Howard Gardner: la teoría de las inteligencias múltiples. http://materialestic.es/transicion/apuntes/Los.8.tipos.de.inteligencia.segun.Howard.Gardner.pdf

Monje, C. (2011). Metodología de la investigación cuantitativa y cualitativa. Guía didáctica. Universidad Sur Colombiana.

Palacios, M. (2008). El enfoque comunicativo en la construcción de textos expositivos en estudiantes de la Facultad de Educación de la Universidad Nacional del Centro del Perú [Tesis de Doctorado, Universidad Nacional del Centro del Perú].

Palenzuela, M. (2012). Inteligencias múltiples y aprendizaje del inglés en el aula de adultos mayores [Tesis de Doctorado, Universidad de Almería]. http://www.diegomarin.net/ ual/es/ensenanza-y-aprendizaje-de-lenguas/662-inteligencias-multiples-y-aprendizaje-del-ingles-9788416027491.html

Perea, K. (2017). Lengua extranjera a través de las inteligencias múltiples y su relación con la inteligencia emocional [Tesis de maestría, Universidad de La Laguna]. Repositorio Institucional, Universidad de La Laguna. https://riull.ull.es/xmlui/handle/915/5195

Ramiro Sáez, E. (2017). Adquisición de la lengua extranjera inglés por medio de las inteligencias múltiples [Tesis de máster, Universidad de Valladolid]. Repositorio Institucional de la Universidad de Valladolid. http:/uvadoc.uva.es/handle/10324/27000

Sánchez, H. y Reyes, C. (2006). Metodología y diseños de la investigación científica. Visión Universitaria.

Sánchez, H. y Reyes, C. (2009). Metodología de la Investigación y Diseños en la Investigación Científica. Visión Universitaria.

Torres, D. y Valdivieso, J. (2013). Aprendizaje con base en tareas e inteligencias múltiples dominantes de los estudiantes de $9^{\circ}$ grado del Colegio de Bachillerato Universidad Libre para el fortalecimiento de su competencia comunicativa en inglés [Tesis de licenciatura, Universidad Libre de Colombia]. https://repository.unilibre.edu.co/ bitstream/handle/10901/7923/TorresRinconDiegoFelipe2013.pdf;sequence=1 